\title{
A REMARK ON THE GROTHENDIECK RESIDUE MAP'
}

\author{
JAMES B. CARRELL
}

\begin{abstract}
The purpose of this note is to give a direct proof that a global integral over a compact complex manifold $X$ can be evaluated on the zero set of a meromorphic vector field on $X$ with isolated zeros via a Grothendieck residue morphism. A special case of this evaluation is the meromorphic vector field theorem of Baum and Bott [1]. The present proof suggests some complements of the M.V.F. Theorem which are contained in Theorem 2.
\end{abstract}

1. Introduction. Statement of results. Given a finite, nontrivial, but possibly unreduced subvariety $Z$ of a connected compact complex manifold $X$ of dimension $n$, and given $\omega \in H^{n}\left(X, \Omega^{n}\right)$, the global integral $\int_{X} \omega$ can always be evaluated as a sum of residues (in the sense of Grothendieck) on $Z$. The proofs of this fact in the literature are valid in algebraic geometry and are necessarily complicated, cf. [7], [13]. On the other hand, what we shall show is that if one makes the assumption that $Z$ is the variety of zeros of a meromorphic vector field $V$ on $X$, then a reformulation of the fundamental commutative diagram of [13], which makes the contribution from $Z$ explicit, can be proven simply (Theorem 1). Moreover this approach immediately suggests some complements to the Meromorphic Vector Field Theorem [1], [2], which are given in Theorem 2.

To state our results precisely, let $T$ denote the holomorphic tangent bundle of $X, L$ a holomorphic line bundle on $X$, and $\Theta$ (resp. $\mathcal{L}$ ) the sheaf of germs of holomorphic sections of $T$ (resp. $L$ ). Contraction by $V \in H^{0}(X, T \otimes L)$ on holomorphic $p$-forms is an operator $i(V): \Omega^{p} \rightarrow \Omega^{p-1} \otimes \mathcal{L}$ on the sheaf level, which defines a sheaf of ideals $I_{Z}=i(V)\left(\Omega^{1} \otimes \mathcal{L}^{-1}\right)$ in $\Omega^{0}=0$. The subvariety $Z$ defined by $I_{Z}$ is called the variety of zeros of $V$. The structure sheaf of $Z$ is by definition the sheaf of rings $\theta_{z}=\theta / I_{z}$. For any sheaf $\mathcal{F}$ of $\theta$-modules on $X$, let $\mathscr{F}_{Z}=\mathscr{F} \otimes_{\mathcal{\theta}} \theta_{Z}$. The Grothendieck Residue Map we will associate to $V$ is a map Res: $H^{0}\left(X, \mathfrak{L}_{Z}^{n}\right) \rightarrow C$ about which we shall prove the following result. It will always be assumed that $Z$ is finite but nontrivial.

THeOREM 1. There exists a map $m: H^{0}\left(X, \mathfrak{L}_{Z}^{n}\right) \rightarrow H^{n}\left(X, \Omega^{n}\right)($ depending only on $V$ ) such that

Received by the editors May 28, 1976 and, in revised form, April 20, 1977.

AMS (MOS) subject classifications (1970). Primary 32A25, 14F25.

'Supported in part by a grant from the National Research Council. 


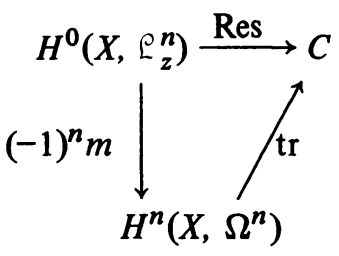

commutes, where tr is the map $(1 / 2 \pi i)^{n} \int_{X}(c f .[13])$.

Given an ad-invariant linear map $p: \operatorname{gl}(n, C)^{\otimes k} \rightarrow C$, there is a natural element $p\left(V_{0}\right)$ of $H^{0}\left(X, \mathcal{L}_{Z}^{k}\right)$ such that in the case $k=n, \operatorname{tr}\left(m\left(p\left(V_{0}\right)\right)\right)$ is a characteristic number of the virtual bundle $T-L^{-1}$. The assertion $(-1)^{n} \operatorname{tr}\left(m\left(p\left(V_{0}\right)\right)\right)=\operatorname{Res} p\left(V_{0}\right)$ is of course the M.V.F. Theorem. In $\S 5$, we will show that in addition one can use (1) to prove

TheOREM 2. Suppose $\operatorname{deg} p=k<n$ and that $\sigma \in H^{0}(X, L)$. Let $\sigma^{n-k} p\left(V_{0}\right)$ denote the image of $\sigma^{n-k} \otimes p\left(V_{0}\right)$ under the natural pairing $H^{0}\left(X, L^{n-k}\right) \otimes$ $H^{0}\left(X, \mathcal{L}_{Z}^{k}\right) \rightarrow H^{0}\left(X, \mathcal{L}_{Z}^{n}\right)$. Then $\operatorname{Res} \sigma^{n-k} p\left(V_{0}\right)=0$.

To prove Theorem 1, we employ a double complex with differentials $i(V)$ and $\bar{\partial}^{-}$to compute $H^{0}\left(X, \mathcal{L}_{Z}^{n}\right)$. The mapping $m$ is an edge morphism in this double complex. (1) is a consequence of the projector trick of Bott [3] and an interesting local integral representation formula for Res (Lemma 4), which simply amounts to combining a local I.R.F. for the partial derivatives of a holomorphic function at a point with the computational algorithm for the local residue involving the Nullstellensatz.

2. A double complex. For a fixed integer $m$, let $A^{p, q}\left(L^{m}\right)$ denote $C^{\infty}$, $L^{m}$-valued forms on $X$ of type $(p, q)$. The operators $i(V): A^{p, q}\left(L^{m}\right) \rightarrow$ $A^{p-1, q}\left(L^{m+1}\right)$ and $\bar{\partial}: A^{p, q}\left(L^{m}\right) \rightarrow A^{p, q+1}\left(L^{m}\right)$ satisfy $i(V)^{2}=\bar{\partial}^{2}=\overline{\partial i}(V)+$ $i(V) \bar{\partial}=0$, so $D=i(V)+\bar{\partial}$ is a total differential for the complex $C_{m}^{k}=$ $\Sigma_{p} C_{m}^{p, k-p}$ formed from the double complex $\left\{C_{m}^{p, q}=A^{p, q}\left(L^{m-p}\right), i(V), \bar{\partial}\right\}$.

LEMMA 1. Assuming that $Z$ is finite, then $H^{0}\left(C_{m}^{\cdot}\right) \cong H^{0}\left(X, \complement_{Z}^{m}\right)$.

Let $\mathcal{E}^{p, q}\left(\mathfrak{L}^{k}\right)$ denote the sheaf of germs of $C^{\infty}, L^{k}$-valued $(p, q)$ forms on $X$. To prove Lemma 1 , we need

LEMMA 2. For any $k$ we have a fine resolution of $\mathcal{L}_{Z}^{k}$ :

$$
\begin{aligned}
0 & \rightarrow \mathcal{L}_{Z}^{k} \stackrel{h}{\rightarrow} \mathcal{E}^{0,0}\left(\mathcal{L}^{k}\right) / i(V) \mathcal{E}^{1,0}\left(\mathcal{L}^{k-1}\right) \stackrel{\bar{\partial}}{\rightarrow} \mathcal{E}^{0,1}\left(\mathcal{L}^{k}\right) / i(V) \mathcal{E}^{1,1}\left(\mathcal{L}^{k-1}\right) \\
& \stackrel{\bar{\jmath}}{\rightarrow} \cdots \stackrel{\bar{\partial}}{\rightarrow} \mathcal{E}^{0, n}\left(\mathcal{L}^{k}\right) / i(V) \mathcal{E}^{1, n}\left(\mathcal{L}^{k-1}\right) \rightarrow 0
\end{aligned}
$$

where $h$ is the natural inclusion.

Proof. One first notes that for any $k$ the Dolbeault resolution

$$
0 \rightarrow \mathcal{L}^{k} \rightarrow \mathcal{E}^{0,0}\left(\mathcal{L}^{k}\right) \stackrel{\bar{\partial}}{\rightarrow} \mathcal{E}^{0,1}\left(\mathcal{L}^{k}\right) \rightarrow \cdots \rightarrow \mathcal{E}^{0, n}\left(\mathfrak{L}^{k}\right) \rightarrow 0
$$

is an exact sequence of $\theta$-modules from which (2) is obtained via tensorisation by $\theta_{z}$. Lemma 2 follows from the fact that $\mathcal{E}^{p, q}\left(\complement^{k}\right)$ is a flat $\theta$-module, 
by the Malgrange Preparation Theorem [9].

This lemma does not assume $Z$ is finite. Finiteness of $Z$ is used to conclude exactness of

$$
0 \rightarrow \Omega^{n} \otimes \mathcal{L}^{k-n} \stackrel{i(V)}{\rightarrow} \Omega^{n-1} \otimes \mathfrak{L}^{k-n+1} \rightarrow \cdots \rightarrow \Omega^{1} \otimes \mathcal{L}^{k-1} \stackrel{i(V)}{\rightarrow} \mathfrak{L}^{k} .
$$

To prove Lemma 1, consider the exact sequence obtained from (2):

$$
0 \rightarrow H^{0}\left(X, \mathcal{L}_{Z}^{k}\right) \stackrel{h}{\rightarrow} C_{k}^{0,0} / i(V) C_{k}^{1,0} \stackrel{\bar{\partial}}{\rightarrow} C_{k}^{0,1} / i(V) C_{k}^{1,1}
$$

For $s \in H^{0}\left(X, \varrho_{Z}^{k}\right)$, choose $s_{0} \in C_{k}^{0,0}$ such that $h(s)=s_{0}$ modulo $i(V) C_{k}^{1,1}$. By (4), $\bar{\partial} s_{0}=i(V) s_{1}$ for some $s_{1} \in C_{k}^{1,1}$. Since $i(V) \bar{\partial} s_{1}=-\bar{\partial} i(V) s_{1}=\bar{\partial} s_{0}=$ 0 , there exists by (3) an $s_{2} \in C_{k}^{1,2}$ so that $\bar{\partial} s_{1}=i(V) s_{2}$. Continuing in this manner, one gets a total cocycle $S=s_{0}-s_{1}+\cdots+(-1)^{n} s_{n}$ of $C_{k}$ of degree 0 , and the map $s \rightarrow S$ induces a morphism $\phi_{k}: H^{0}\left(X, \mathcal{L}_{Z}^{k}\right) \rightarrow H^{0}\left(C_{k}^{-}\right)$. To show that $\phi_{k}$ is an isomorphism, we produce its inverse. Let $S$ be a total cocycle in $C_{k}^{0}$ denoted as above. Then $\bar{\partial} s_{0}$ is in $i(V) C_{k}^{1,1}$. Hence by (4), there exists a unique $s \in H^{0}\left(X, \mathcal{L}_{Z}^{k}\right)$ such that $h(s)=s_{0} \bmod i(V) C_{k}^{1,0}$. Then $S \rightarrow s$ induces the inverse of $\phi_{k}$, and the proof of Lemma 1 is complete.

One now defines $m: H^{0}\left(X, \mathcal{L}_{Z}^{n}\right) \rightarrow H^{n}\left(X, \Omega^{n}\right)(n=\operatorname{dim} X)$ by composing $\phi_{k}^{-1}$ with the natural edge morphism $H^{0}\left(C_{n}\right) \rightarrow H^{0}\left(X, \Omega^{n}\right)$ induced by mapping $S$ in $C_{n}^{0}$ to the component $s_{n}$ of $S$ in $A^{n, n}\left(L^{9}\right)$.

3. The morphism Res. Let $U$ be an open ball about the origin $O$ of $C^{n}$, which is the only common zero of $a_{1}, \ldots, a_{n} \in H^{0}(U, \theta)$. The local residue at $O$ of $\omega \in H^{0}\left(U, \Omega^{n}\right)$ with respect to $a_{1}, \ldots, a_{n}$ is defined in [11] as

$$
\operatorname{Res}\left(a_{1} \cdots a_{n}\right)=\left(\frac{1}{2 \pi i}\right)^{n} \int_{\partial D \times \cdots \times \partial D} \frac{\omega}{a_{1} \cdots a_{n}}
$$

where $D$ is a disc about $O$ in $C$ chosen so that $\partial D \times \cdots \times \partial D$ misses the hypersurface $\left\{z:\left(a_{1} \cdots a_{n}\right)(z)=0\right\}$ in $U$. The local residue defined by (5) coincides with the Grothendieck residue, and hence can be computed by the well-known algorithm [1], [2], [7], [11], [13]. If $a_{1}, \ldots, a_{n} \in H^{0}(U, \mathcal{L})$, then

$$
\operatorname{Res}\left(a_{1} \cdots a_{n}\right)
$$

is unambiguously defined for all $\omega \in H^{0}\left(U, \Omega^{n} \otimes \mathfrak{L}^{n}\right)$.

Suppose $V \in H^{0}(X, T \otimes L)$ has isolated zeros $Z$, and let $\left(z_{1}, \ldots, z_{n}\right)$ denote local coordinates for $X$ near $\zeta \in Z$ with $z_{i}(\zeta)=0$ for each $i$. One may locally express $V=\sum a_{i} \partial / \partial z_{i}$, where $a_{1}, \ldots, a_{n}$ are local sections of $L$ whose only common zero is $\zeta$. Given $s \in \mathcal{L}_{\zeta}^{n}$, the local residue of $s$ at $\zeta$ is defined to be

$$
\operatorname{Res}(s)_{\zeta}=\operatorname{Res}\left(\begin{array}{c}
s d z_{1}, \ldots d z_{n} \\
a_{1}, \ldots, a_{n}
\end{array}\right)
$$

Under change of coordinates, Res transforms in a manner so as to imply that (6) depends only on $V$ and $s$. In fact, $\operatorname{Res}(s)_{\zeta}=0$ if $s \in\left(I_{Z} \mathcal{L}^{n}\right)_{\zeta}$ (by [7]), and consequently Res is defined as a morphism $\operatorname{Res}_{5}: \mathcal{L}_{Z, 5}^{n} \rightarrow C$. Define Res: $H^{0}\left(X, \rho_{Z}^{n}\right) \rightarrow C$ by $\operatorname{Res}=\Sigma_{Z} \operatorname{Res}_{\zeta}$. 
4. The proof of Theorem 1. In order to localize $\omega=m(S) \in H^{n}\left(X, \Omega^{n}\right)$, we will use Bott's projector trick. Recall that projector for $V$ is an $L^{-1}$ valued $(1,0)$ form $\pi$ on $X-Z$ such that $\pi(V)=1$. Bott's key observation concerning projectors can be rephrased in this context as the observation that the differential form

$$
\tau=\pi\left(s_{0}(\bar{\partial} \pi)^{n-1}-s_{1}(\bar{\partial} \pi)^{n-2}+\cdots+(-1)^{n-1} s_{n-1}\right)
$$

on $X-Z$ satisfies $s_{n}=(-1)^{n-1} d \tau$ provided $S=s_{0}+s_{1}+\cdots+s_{n}$ is a total cocycle of $C_{n}^{0}$. If $\left\{W_{j}\right\}$ is a finite covering of $Z$ by disjoint coordinate balls such that $W_{i} \cap Z=\left\{\zeta_{i}\right\}$, then by Stokes' Theorem

$$
\int_{X} s_{n}=(-1)^{n} \sum \int_{\partial W_{j}} \tau
$$

Now the right-hand side of (8) can be vastly simplified by the following observation.

Lemma 3. For any $\delta \in H^{0}(C)$, there is a representing cocycle $S=s_{0}$ $+\cdots+s_{n}$ such that $s_{0}$ is holomorphic near $Z$, and, if $i>0$, then $s_{i}=0$ near $Z$.

Proof. $s_{0}$ can obviously be so chosen. The existence of the $g_{i}$ follows from repeated application of (3).

The proof of Theorem 1 now results from the following local integral representation formula for Res.

LEMMA 4. For any projector $\pi$ for $V$ and for any $\zeta \in Z$,

$$
\operatorname{Res}(s)_{\zeta}=\left(\frac{1}{2 \pi i}\right)^{n} \int_{\partial W} s \pi(\bar{\partial} \pi)^{n-1}
$$

where $s \in \mathfrak{L}_{\zeta}^{n}$ and $W$ is a sufficiently small ball centered at $\zeta$.

Proof. Since $Z$ is finite, $\mathfrak{L}_{Z, 5}^{n} \subset H^{0}\left(X, \mathfrak{L}_{Z}^{n}\right)$. Therefore, $m(s)$ is defined and $\int_{X} m(s)=\int_{\partial W} s \pi(\bar{\partial} \pi)^{n-1}$. Hence (9) is independent of $\pi$. Let $\pi$ be the projector for $V$ defined in a neighborhood $W$ of $\zeta$ as follows. Let $V=\Sigma a_{i} \partial / \partial z_{i}$ on $W$ as above. By Hilbert's Nullstellensatz, there exist positive integers $\alpha_{1}, \ldots, \alpha_{n}$ and $b_{i j} \in H^{0}\left(W, L^{-1}\right)$ such that $z_{i}^{\alpha_{i}}=\Sigma b_{i j} a_{j}$. Set $\pi=$ $u^{-1} \sum \bar{z}_{i}^{\alpha_{i}} b_{i j} d z_{j}$, where $u=\Sigma\left(z_{i} \bar{z}_{i}\right)^{\alpha_{i}}$. Note

$$
\begin{aligned}
& \pi(\bar{\partial} \pi)^{n-1}=(n-1) !(-1)^{n(n-1) / 2} u^{-n} \operatorname{det}\left\|b_{i j}\right\| \\
& \quad \times \sum(-1)^{i-1} \bar{z}_{i}^{\alpha_{i}} d \bar{z}_{1}^{\alpha_{1}} \wedge \cdots \wedge \widehat{d \bar{z}_{i}^{\alpha_{i}}} \wedge \cdots \wedge d \bar{z}_{n}^{\alpha_{n}} \wedge d z_{1} \wedge \cdots \wedge d z_{n} .
\end{aligned}
$$

Now, by applying a standard I.R.F. for the partial derivatives of a holomorphic function $g$ defined in a neighbourhood of $\bar{W}[10$, p. 56], one gets

$$
\left(\frac{1}{2 \pi i}\right)^{n} \int_{\partial W} g \pi(\bar{\partial} \pi)^{n-1}=1 /(\alpha-1) ! D^{\alpha}\left(\operatorname{det}\left\|b_{i j}\right\| g\right)(\zeta)
$$

where $\alpha$ and $\alpha-1$ denote, respectively, the multi-indices $\left(\alpha_{1}, \ldots, \alpha_{n}\right)$ and $\left(\alpha_{1}-1, \ldots, \alpha_{n}-1\right)$. Lemma 4 follows from the fact that the r.h.s. of $(10)$ is 


$$
\operatorname{Res}_{\zeta}\left(\begin{array}{c}
g d z_{1}, \ldots d z_{n} \\
a_{1}, \ldots, a_{n}
\end{array}\right)
$$

by the algorithm.

To finish the proof of (1), let $s \in H^{0}\left(X, \mathcal{L}_{Z}^{n}\right)$ and let $S=s_{0}+s_{1}+\cdots+$ $s_{n}$ be a total cocycle of $C_{n}^{0}$ representing $\phi(s)$ satisfying all the conclusions of Lemma 3. Then $(-1)^{n} m(S)=(-1)^{n} s_{n}$, so

$$
\left(-\frac{1}{2 \pi i}\right)^{n} \int_{X} s_{n}=\left(\frac{1}{2 \pi i}\right)^{n} \sum \int_{\partial W_{j}} s_{0} \pi(\bar{\partial} \pi)^{n-1}=\operatorname{Res}(s)
$$

by (9) and Lemma 4.

5. Some residue formulas. If $V$ is a global section of the tangent sheaf $\Theta$ of $X$ (i.e. a holomorphic vector field on $X$ ), then the Lie bracket $Y \rightarrow[V, Y]$ induces a $C$-linear map $\tilde{V}: \Theta \rightarrow \Theta$ lifting the derivation $V: \theta \rightarrow \theta$, i.e. $\tilde{V}(f Y)=V(f) Y+f \tilde{V}(Y)$. Any sheaf $\mathcal{F}$ of $\theta$-modules admitting such a lifting $\tilde{V}$ is called $V$-equivariant and $\tilde{V}$ is called an equivariant lift of $V$. Note that $\tilde{V}$ defines $V_{0} \in H^{0}\left(X, \operatorname{Hom}_{\Theta}(\Theta, \Theta)_{z}\right)$. Analogously, an equivariant lift $\tilde{V}: \quad \mathscr{F} \rightarrow \mathscr{F} \otimes L$ of $V \in H^{0}(X, T \otimes L)$ defines an element $V_{0} \in$ $H^{0}\left(X,\left(\operatorname{Hom}_{\Theta}(\mathscr{F}, \mathscr{F}) \otimes \mathcal{L}\right)_{Z}\right)$. Now $\Theta$ is generally not equivariant for an arbitrary $V \in H^{0}(X, T \otimes L)$, however $\tilde{V}$ admits a well-defined localization $V_{0} \in H^{0}\left(X,\left(\operatorname{Hom}_{\Theta}(\Theta, \Theta) \otimes \mathcal{E}\right)_{z}\right)$. In fact, choose a covering $\left\{U_{\alpha}\right\}$ of $X$ such that both $L \mid U_{\alpha}$ and $T \mid U_{\alpha}$ are locally trivial for each $\alpha$, and on $U_{\alpha}$ write $V=w_{\alpha} \otimes t_{\alpha}$ where $w_{\alpha} \in H^{0}\left(U_{\alpha}, \Theta\right), t_{\alpha} \in H^{0}\left(U_{\alpha}, \mathcal{L}\right)$, and $t_{\alpha}$ is nowhere vanishing. For $Y \in H^{0}\left(U_{\alpha}, \Theta\right)$, set $\tilde{V}_{\alpha}(Y)=\left[w_{\alpha}, Y\right] \otimes t_{\alpha}$. Note that on $U_{\alpha}$ $\cap U_{\beta}, \tilde{V}_{\beta}(Y)-\tilde{V}_{\alpha}(Y)=t_{\beta} t_{\alpha}^{-1} i(Y) d\left(t_{\beta} t_{\alpha}^{-1}\right) \otimes V$, consequently the $\tilde{V}_{\alpha}$ patch on $Z$ giving $V_{0}$ as asserted.

Let $p\left(V_{0}\right)$ denote the element of $H^{0}\left(X, \varrho_{Z}^{k}\right)$ obtained by applying the ad-invariant symmetric linear map $p: \operatorname{gl}(n, C)^{\otimes k} \rightarrow C$ to $V_{0}$. In order to construct the class $\phi_{k}\left(p\left(V_{0}\right)\right)$ in $H^{0}\left(C_{k}\right)$, first choose a local holomorphic connection $D_{\alpha}$ for $T \mid U_{\alpha}$, and let $D=\Sigma \rho_{\alpha} D_{\alpha}$ be the connection of type $(1,0)$ on $T$ where $\rho_{\alpha}$ is a partition of unity fitted to $\left\{U_{\alpha}\right\}$. Following [1], consider the $\theta$-linear map $\psi_{\#}: \Omega^{1} \rightarrow \operatorname{Hom}(\Theta, \Theta) \otimes \Omega^{1}$ so that if $\omega \in \Omega_{x}^{1}$, and $Y \in \Theta_{x}$ then $i(Y) \psi_{\#} \omega$ is the element of $\operatorname{Hom}\left(\Theta_{x}, \Theta_{x}\right)$ such that $i(Y) \psi_{\#} \omega(w)=$ $\omega(w) Y$. Let $\Gamma \in A^{1,0}(\operatorname{Hom}(T, T))$ be given by $\Gamma=\Sigma \rho_{\alpha}\left\{\psi_{\#}\left(t_{\alpha}^{-1} d t_{\alpha}\right)-D_{\alpha}\right\}$, and let $K^{*}=\bar{\partial} \Gamma \in A^{1,1}(\operatorname{Hom}(T, T))$. Finally, define $\tau=\Sigma \rho_{\alpha}\left\{\tilde{V}_{\alpha}-i(V) D_{\alpha}\right\}$ $\in A^{0,0}(\operatorname{Hom}(T, T) \otimes L)$. The following lemma is proved by a local calculation which will be omitted.

LEMMA 5. $i(V) K^{*}+\bar{\partial} \tau=0$ in $A^{0,1}(\operatorname{Hom}(T, T) \otimes L)$.

One may therefore perform the ad-invariant map construction to get a class $p\left(K^{*}+\tau\right)=p\left(\left(K^{*}+\tau\right)^{\otimes k}\right)$ in $C_{k}^{0}$ where $k=\operatorname{deg} p$. Because of Lemma 5, it follows that $p\left(K^{*}+\tau\right)$ is a total cocycle. Note

$$
p\left(K^{*}+\tau\right)=p\left(K^{* \otimes k}\right)+k p\left(K^{* \otimes k-1} \otimes \tau\right)+\cdots+p\left(\tau^{\otimes k}\right) .
$$

Note that by definition, $p\left(\tau^{\otimes k}\right)$ is an extension of $p\left(V_{0}\right)$ to $X$. If $k=\operatorname{deg} p=$ 
$n$, we therefore get by (1) that

$$
\left(\frac{1}{2 \pi i}\right)^{n} \int_{X} p\left(K^{* \otimes n}\right)=\operatorname{Res} p\left(V_{0}\right)
$$

which is part of the M.V.F. Theorem. The rest of the theorem is an identification of $(1 / 2 \pi i)^{n} \int_{X} p\left(K^{* \otimes n}\right)$ with a characteristic number of $T-$ $L^{-1}$.

In order to prove Theorem 2 , note that in the case $\operatorname{deg} p=k<n$, if $\sigma \in H^{0}(X, L)$, then $\sigma^{n-k} p\left(V_{0}\right)$ lies in $H^{0}\left(X, \mathcal{L}_{Z}^{n}\right)$ and $m \phi_{n}\left(\sigma^{n-k} p\left(V_{0}\right)\right)=$ $(-1)^{n} m\left(\sigma^{n-k} \times p\left(K^{* \otimes k}\right)\right)$ since $\phi_{n}\left(\sigma^{n-k} p\left(V_{0}\right)\right)$ is represented by the total cocycle $\sigma^{n-k} p\left(K^{*}+\tau\right)$ of $C_{n}^{0}$ (due to the fact that $\sigma^{n-k}$ commutes with both $i(V)$ and $\bar{\partial})$. But clearly $m\left(\sigma^{n-k} p\left(K^{*}+\tau\right)\right)=0$ since $\operatorname{deg} p<n$. Consequently $\operatorname{Res}\left(\sigma^{n-k} p\left(V_{0}\right)\right)=0$, by (1), as asserted.

REMARK. The notion of $V$-equivariance is studied in [1] and in [4] from a different viewpoint. In [4] it is shown that if $X$ is projective, then given a holomorphic vector bundle $E$ on $X$, there exists a $V \in H^{0}(X, T \otimes L)$ with isolated zeros for which $E$ is $V$ equivariant. Thus $E$ 's characteristic numbers can be computed as above.

\section{REFERENCES}

1. P. Baum and R. Bott, On the zeros of meromorphic vector fields, Essays on Topology and Related Topics (Mémoires dédiés à Georges de Rham), Springer, New York, 1970, pp. $29-47$.

2. __ Singularities of holomorphic foliations, J. Differential Geometry 7 (1972), 279-342.

3. R. Bott, Vector fields and characteristic numbers, Michigan Math. J. 14 (1967), 231-244.

4. J. B. Carrell and D. Lieberman, Vector fields and Chern numbers, Math. Ann. 225 (1977), 223-233.

5. S. S. Chern, Meromorphic vector fields and characteristic numbers, Scripta Math. 29 (1973), 243-252.

6. W. Koppelman, The Cauchy integral for differential forms, Bull. Amer. Math. Soc. 73 (1967), 554-556.

7. R. Hartshorne, Residues and duality, Lecture Notes in Math., Vol. 20, Springer-Verlag, Berlin and New York, 1966.

8. F. R. Harvey, Integral formulae connected by Dolbeault's isomorphism, Rice Univ. Studies 20 (1966).

9. B. Malgrange, Ideals of differentiable functions, Oxford Univ. Press, Oxford, 1966.

10. F. Norguet, Introduction $\dot{a}$ fonctions de plusieurs variables complexes, Lecture Notes in Math., Vol. 409, Springer-Verlag, Berlin and New York, 1974.

11. Y. L. Tong, Integral representation formulae and Grothendieck residue symbol, Amer. J. Math. 95 (1973), 904-917.

12. De Rham's integrals and Lefschetz fixed point formula for $d^{\prime \prime}$ cohomology, Bull. Amer. Math. Soc. 78 (1972), 420-422.

13. J. L. Verdier, Base change for twisted inverse image of coherent sheaves, Algebraic Geometry, Oxford Univ. Press, London, 1969, pp. 393-408.

Departiment of Mathematics, University of British Columbia, Vancouver, Canada 